BULLETIN 13 (2), 1970

\title{
THESIS ABSTRACTS
}

Applications of the Theory of Categories to Analysis. By JOAN M. NEGREPONTIS, MCGill University, APrIL 1970. (Supervisor: J. Lambek.)

Let $A, K, C, A b, B, S$ denote the categories of commutative $C^{*}$-algebras with identity, compact Hausdorff spaces, compact abelian groups, abelian groups, commutative von Neumann algebras with identity, sets, respectively. The Gelfand duality between $A$ and $K$ is proved by means of the theory of triples. Central in this proof are the tripleability of $A$ over $S$ with respect to the unit disc functor and the tripleability of $K^{\mathrm{op}}$ over $S$ with respect to the functor $K(-, I)$, where $I$ is the unit disc of the complex numbers. The Pontryagin duality between $C$ and $A b$ is proved similarly. $B$ is characterized as a subcategory of $A$ by means of a functor from $B$ to $K$ with which $B$ is shown to be tripleable. The big group algebra functor from $A b$ to $B$ is studied, revealing the co-tripleability of $A b$ over the subcategory of $B$ consisting of Hopf-von Neumann algebras.

Non-Linear Theories in Two-Dimensional Fully and Partially Cavitating Flow. By K. M. Agrawal, University of Windsor, June 1969. (Supervisor: A. C. Smith.)

The single-spiral vortex model proposed by Tulin (1964) is used to represent a finite two-dimensional cavity. For flow past a symmetric wedge in the absence of gravity, it is found to possess the same first-order characteristics as the modified Riabouchinsky and re-entrant jet models, and as the usual linear theory.

Secondly, cavitating flow past a symmetric wedge in the presence of a transverse gravity field, at infinite depth, is considered. The spiral vortex model is again used, and the Riemann-Hilbert method applied. Numerical integration shows that a finite cavity in the gravity case lies entirely inside the position of the cavity when gravity is absent. The effect of gravity on the drag coefficient is found to be small, and to increase with decreasing cavitation number.

Lastly, fully and partially cavitating flows past a flat plate are considered using Wu's model (1962), but with different conditions. The Riemann-Hilbert method is used, and results obtained for lift and drag coefficients which show a smooth transition from partially to fully cavitating flow, in contradistinction to linear theories. Furthermore the drag coefficient is found to be positive and to increase with cavity number, while in Wu's theory it decreases in the partially cavitating regime and eventually becomes negative. For large values of cavitation number the model used does not predict maximum lift nor does it yield the fully wetted limit. 